\section{Fatores psicossociais e socioeconômicos
relacionados à insônia e menopausa: \\ Fatores psicossociais e socioeconômicos
relacionados à insônia e menopausa: Estudo Pró-Saúde}

\author{
Psychosocial and socioeconomic factors related to \\ insomnia and menopause: Pró-Saúde Study
}

\author{
Factores psicosociales y socioeconómicos \\ relacionados con el insomnio y la menopausia: \\ Estudio Pro-Salud \\ Estudio Pro-Salud
}




\section{Introdução}

Apesar de a insônia ser um dos sintomas mais frequentemente associados à menopausa, sua etiologia ainda é controversa. Estudos se referem à dificuldade em determinar se as alterações no sono iniciadas na meia-idade são devidas ao envelhecimento ou ao estado de menopausa 1,2. Embora a insônia apresente alta prevalência nesse período da vida, ela é frequentemente negligenciada pelos médicos e pelas mulheres que não a reconhecem como um problema de saúde, apesar da deterioração da qualidade do sono ${ }^{3,4}$.

Alguns estudos relatam que a insônia na menopausa está mais fortemente associada aos sintomas vasomotores, provavelmente decorrentes da cascata de sintomas, que inclui fogacho e sudorese noturnos 5,6. Outros demonstram que a insônia também pode estar associada a sintomas psicológicos, o que refletiria a sua associação com depressão 1,6 e com altos níveis de estresse 7,8 .

O conhecimento de que componentes psicológicos, tanto positivos quanto negativos, podem afetar o risco para desfechos em saúde tem crescido ${ }^{9}$, embora as evidências empíricas sejam escassas, sendo poucos os dados sobre fatores psicológicos e insônia 10,11. Estudos com base em diagnósticos psiquiátricos, padrões de personalidade e eventos de vida produtores de estresse têm investigado fatores emocionais envolvidos no desenvolvimento e na persistência de insônia 12 .

Aspectos ligados à depressão 2,10,13 e à ansiedade 10 são frequentemente relacionados à menopausa e à insônia, mas os resultados não são consistentes. Embora a maioria dos estudos demonstre um aumento dos níveis de ansiedade $\mathrm{e}$ depressão durante a peri e pós-menopausa 14,15, outros não demonstram diferenças 10,16.

Eventos de vida produtores de estresse também estão fortemente associados com a insônia crônica 17 , sendo frequentemente mediados por fatores de personalidade. Os insones tendem a ser mais descontentes, ter menos satisfação em relacionamentos interpessoais e baixa autoestima, tendo mecanismos de manejo inadequados para lidar com o estresse 12. A combinação de vulnerabilidade psiquiátrica e os eventos de vida produtores de estresse estabelecem vários links causais entre os sintomas da menopausa e transtornos psiquiátricos. Mulheres na menopausa que sofreram eventos estressores, como a morte de parente próximo, doença ou crise no casamento, têm altos escores de morbidade psicológica e exacerbação dos sintomas que acompanham a menopausa 16,18 .
O presente artigo tem como principais objetivos investigar a prevalência de insônia, avaliar sua associação com o estado de menopausa e verificar se tal associação é independente de variáveis socioeconômicas e demográficas (idade, renda, escolaridade), que podem atuar como potenciais variáveis de confundimento, e de circunstâncias psicossociais (transtornos mentais comuns, falta de apoio social e eventos de vida produtores de estresse), que são potenciais mediadoras nessa relação.

\section{Métodos}

Desenho e população de estudo

Este estudo transversal faz parte da Fase 1 (1999) do Estudo Pró-Saúde cujo objetivo geral é investigar o papel de determinantes biológicos e socioculturais nos padrões de saúde e de morbidade entre funcionários técnicos administrativos de campi universitários no Estado do Rio de Janeiro, Brasil.

A população alvo do Estudo Pró-Saúde em 1999 foi composta por todos os funcionários técnicos administrativos da universidade, excluindo os cedidos, aposentados ou licenciados ( $\mathrm{n}=$ 4.448). Após a exclusão das recusas, licenciados e dos funcionários não localizados, a população entrevistada foi de 4.030 funcionários (90,6\% da população alvo). A população feminina participante na Fase 1 do Estudo Pró-Saúde foi de 2.238. No presente estudo, foram consideradas, para análise, aquelas com respostas completas às perguntas sobre insônia e menopausa, totalizando uma população de estudo de 2.190 mulheres.

\section{Instrumentos}

Foi utilizado um questionário multidimensional autopreenchível, aplicado por aplicadores treinados 19, no qual foram incluídas perguntas relativas às exposições principais apresentadas neste estudo, bem como todas as covariáveis.

\section{- Variável independente}

\section{a) Menopausa}

O tempo de amenorreia foi calculado diminuindo-se a idade da menopausa referida pela mulher da sua idade no dia da entrevista. Entretanto, a classificação final também levou, em consideração, a resposta dada à pergunta: "Por que você não menstrua mais?” cujas possibilidades de resposta eram: (1) menopausa natural; (2) cirurgia para retirada de útero ou ovários; (3) outros trata- 
mentos (hormônios, quimioterapia ou radiação); e (4) outra razão - especificar. Para a análise dos dados, foi criada uma variável politômica para classificação de estado de menopausa, segundo o tempo de amenorreia, na qual foi considerado como "não estando na menopausa" as mulheres com menstruação regular, grávidas ou com menos de 12 meses de amenorreia, em "menopausa recente" as mulheres não grávidas com tempo de amenorreia de 12 a 60 meses, e "menopausa não recente" as mulheres não grávidas com tempo de amenorreia superior a 60 meses.

\section{- Variáveis dependentes}

a) Dificuldade em inciar o sono, dificuldade em manter o sono e queixa geral de insônia

No questionário original, a dificuldade em iniciar o sono foi avaliada a partir da pergunta "Nas últimas duas semanas, com que frequência você teve dificuldade em iniciar o sono?", e a variável dificuldade em manter o sono baseou-se na resposta à pergunta "Nas últimas duas semanas, com que frequência você acordou durante o sono e teve dificuldade para dormir de novo?". A variável "queixa geral de insônia” foi construída como uma combinação dessas duas perguntas. Todas as perguntas tinham cinco opções de resposta tipo likert ("sempre", "quase sempre”, “às vezes", "raramente" e "nunca").

A avaliação da insônia se baseou em critérios de frequência, que são os mais utilizados na literatura 20. Para fins de análise do presente estudo, as variáveis foram categorizadas em "queixa frequente", quando o respondente marcava "sempre" e/ou "quase sempre" em, pelo menos, uma das perguntas; "queixa ocasional”, quando respondia "às vezes" às duas questões relativas à insônia e "ausência de queixa", quando respondia "raramente" e/ou "nunca" às duas perguntas ou "às vezes" a uma das questões e "quase nunca" ou "nunca" a outra.

\section{- Covariáveis}

\section{a) Transtorno mental comum}

Para avaliar a presença de transtorno mental comum, foi utilizado um instrumento para rastreamento, o General Health Questionnaire (GHQ-12) 21, na sua versão resumida, que abrange situações de tensão, depressão, falta de confiança, enfrentamento, incapacidade de luta, insônia de fundo nervoso e outros problemas psicológicos 22. Para cada pergunta, existem 4 possibilidades de respostas (escala tipo likert), cujo ponto de corte considera cada item como presente ou ausente ( 0 ou 1$)$, de acordo com o método do instrumento ${ }^{23}$. No presente estudo, como a variável dependente é "queixa de insônia”, trabalhamos com o questionário retirando da análise a questão sobre o sono (Nas últimas duas semanas, você tem perdido muito sono por preocupação?). Assim, aqueles que foram positivos para 3 itens do GHQ (em 11 itens) foram classificados como casos de transtorno mental comum.

\section{b) Eventos de vida produtores de estresse}

Foram utilizadas perguntas simples e breves que compunham os chamados "eventos de vida produtores de estresse" (do inglês, stressful life events) 24,25 , e o período de referência para a ocorrência do evento foram os 12 meses anteriores ao preenchimento do questionário. As perguntas que compõem o conjunto de eventos de vida produtores de estresse foram agrupadas, e construiu-se uma variável dicotômica, considerando-se como "sim" - ter tido um ou mais eventos de vida produtores de estresse e "não" - não ter tido eventos de vida produtores de estresse durante esse período. Os eventos de vida produtores de estresse avaliados foram: problemas de saúde que impediram a realização de atividades habituais por mais de um mês; internação hospitalar por uma noite, ou mais, em razão de doença ou acidente; falecimento de algum parente próximo; dificuldades financeiras graves; mudança forçada de moradia; rompimento de relação amorosa; assalto ou roubo, mediante uso ou ameaça de violência e ter sido vítima de alguma agressão física 24,25 .

\section{c) Apoio social}

O apoio social foi avaliado pelo conjunto de perguntas utilizadas no Medical Outcomes Study 26 Esse instrumento contém as principais dimensões de apoio social descritas na literatura e apresenta propriedades psicométricas adequadas em sua versão em português 27 . As respostas a essas perguntas foram avaliadas segundo uma escala likert. Depois de obtido o índice padronizado para todas as dimensões, esse escore foi dividido em tercis (alto, médio e baixo apoio social) 27. Para a inclusão dessa covariável nas análises, as categorias foram dicotomizadas em "alto apoio social", para as pessoas cujo escore estava situado na categoria de maior valor (alto apoio social), e "baixo apoio social", para aquelas cujo escore correspondia ao tercil intermediário e inferior (médio e baixo apoio social). 


\section{- Variáveis socioeconômicas e demográficas}

Foram avaliadas as seguintes variáveis socioeconômicas e demográficas: idade, grau de instrução e renda familiar per capita em salários mínimos (SM). A pergunta sobre renda foi originalmente estruturada em faixas de valores expressas em reais, sendo a média dos valores de cada categoria dividida pelo salário mínimo da época, $\mathrm{R} \$$ 136,00. A escolaridade foi categorizada em Ensino Fundamental incompleto, Ensino Fundamental completo/Ensino Médio incompleto, Ensino Médio completo/Ensino Superio incompleto e Ensino Superior completo/pós-graduação.

\section{Análise dos dados}

Na primeira etapa, foi realizada uma descrição geral dos dados pelo exame da distribuição de frequências para cada variável de estudo.

Foram realizadas análises bivariadas para avaliar a associação entre o estado de menopausa e a dificuldade de iniciar o sono, a dificuldade em manter o sono e a queixa geral de insônia. Para avaliar a significância estatística das associações, foram utilizados os testes de qui-quadrado de Pearson.

A análise multivariada foi feita por meio de regressão logística, com cálculo dos odds ratios (OR) simples e ajustados. Foram incluídas, nos modelos, as covariáveis consideradas relevantes, segundo a literatura sobre o tema.

Utilizou-se o programa Epi Info v. 6.0 (Centers for Disease Control and Prevention, Atlanta, Estados Unidos) para a entrada dos dados e o programa Stata 8.0 (Stata Corp., College Station, Estados Unidos) para as análises.

\section{Ética em pesquisa}

A pesquisa foi submetida e aprovada pelo Comitê de Ética em Pesquisa do Hospital Universitário Pedro Ernesto, sob registro no 224/1999. Os participantes assinaram o termo de consentimento livre e esclarecido no momento da coleta de dados, que foi realizada entre agosto e novembro de 1999. Foram tomadas precauções para resguardar a confidencialidade e a qualidade dos dados obtidos, tais como: utilização de bancos de dados diferentes para as respostas do questionário e para os dados de identificação (nome, matrícula e endereço do funcionário - esse guardado sob responsabilidade dos coordenadores da pesquisa), dupla digitação dos dados, certificação e recertificação para os dados antropométricos, entre outras.

\section{Resultados}

Foram entrevistadas 2.238 mulheres na Fase 1 do Estudo Pró-Saúde, e, após a exclusão de 48 questionários com respostas faltantes para as variáveis de exposição (status menopausa) e desfecho (insônia), nossa população de estudo totalizou 2.190 mulheres.

A prevalência da dificuldade de iniciar o sono na população total de mulheres foi de 13,7\% (n = $299)$, sendo a prevalência de $13,5 \%(n=295)$ para a dificuldade em manter o sono e de 16,9\% ( $\mathrm{n}=$ 369) para a queixa geral de insônia.

Na Tabela 1, apresentamos os dados relativos à prevalência da dificuldade em iniciar o sono, da dificuldade em manter o sono e da queixa geral de insônia segundo as variáveis socioeconômicas, demográficas, presença de transtorno mental comum, eventos de vida produtores de estresse e falta de apoio social. Tais associações se mostraram estatisticamente significativas para todas as variáveis do estudo com valor de $\mathrm{p}<0,01$.

As prevalências da dificuldade em iniciar o sono e de manter o sono apresentaram um gradiente tipo dose-resposta em relação às variáveis socioeconômicas e demográficas, sendo diretamente proporcionais à idade e inversamente proporcionais à renda per capita e à escolaridade, tanto para a queixa ocasional como para a frequente.

As prevalências dos três desfechos estudados foram bastante superiores entre as mulheres que sofreram algum evento de vida produtor de estresse nos últimos 12 meses, as que foram classificadas como positivas para transtorno mental comum ou que referiram baixo apoio social, quando comparadas com os respectivos grupos de referência, sendo as diferenças maiores em relação à queixa frequente do que em relação à ocasional.

Nas Tabelas 2 e 3, são descritas as prevalências e respectivos OR brutos e ajustados da associação entre o estado de menopausa e os desfechos estudados. A queixa ocasional teve prevalências maiores que as queixas frequentes para as variáveis dificuldade em iniciar e em manter o sono.

As queixas frequentes (Tabela 2) têm prevalências aumentadas, conforme aumenta o tempo em menopausa, para as três variáveis estudadas, sendo as diferenças estatisticamente significativas ( $\mathrm{p}<0,001)$. As mulheres na menopausa não recente (mais de 60 meses de amenorreia) apresentaram maior prevalência de queixa frequente para dificuldade em manter o sono $(24,8 \%)$ e para queixa geral de insônia (27,5\%). Entre as mulheres em menopausa recente (Tabela 3 ), a 
Prevalência de dificuldade em iniciar o sono, dificuldade em manter o sono e queixa geral de insônia (frequente e ocasional) segundo fatores socioeconômicos, demográficos, presença de transtorno mental comum, evento de vida produtor de estresse e apoio social ( $n=2.190$ ). Estudo Pró-Saúde 1999.

\begin{tabular}{|c|c|c|c|c|c|c|}
\hline & \multicolumn{3}{|c|}{ Queixa ocasional } & \multicolumn{3}{|c|}{ Queixa frequente } \\
\hline & $\begin{array}{c}\text { Dificuldade em } \\
\text { iniciar o sono * } \\
\text { n (\%) }\end{array}$ & $\begin{array}{c}\text { Dificuldade em } \\
\text { manter o sono * } \\
\text { n (\%) }\end{array}$ & $\begin{array}{c}\text { Queixa geral de } \\
\text { insônia * } \\
\text { n (\%) }\end{array}$ & $\begin{array}{c}\text { Dificuldade em } \\
\text { iniciar o sono * } \\
\text { n (\%) }\end{array}$ & $\begin{array}{c}\text { Dificuldade em } \\
\text { manter o sono * } \\
\text { n (\%) }\end{array}$ & $\begin{array}{c}\text { Queixa geral de } \\
\text { insônia * } \\
\text { n (\%) }\end{array}$ \\
\hline \multicolumn{7}{|l|}{ Idade (anos) } \\
\hline Até 39 & $247(24,4)$ & $236(23,3)$ & $158(15,6)$ & $100(9,9)$ & $101(10,0)$ & $137(13,5)$ \\
\hline $40-49$ & $199(24,0)$ & $204(24,7)$ & $141(17,0)$ & $128(15,5)$ & $118(14,2)$ & $143(17,3)$ \\
\hline 50 ou mais & $100(28,6)$ & $99(28,2)$ & $61(17,4)$ & $71(20,3)$ & $76(21,6)$ & $89(25,4)$ \\
\hline \multicolumn{7}{|l|}{ Renda familiar per capita } \\
\hline \multicolumn{7}{|l|}{ (salários mínimos) } \\
\hline Até 3 & $147(30,4)$ & $141(29,2)$ & $90(18,6)$ & $94(19,5)$ & $91(18,9)$ & $114(23,6)$ \\
\hline Entre 3-6 & $203(25,9)$ & $212(27,0)$ & $148(18,9)$ & $86(11,0)$ & $88(11,2)$ & $114(14,5)$ \\
\hline Mais de 6 & $163(20,4)$ & $160(20,0)$ & $103(12,9)$ & $93(11,6)$ & $87(10,9)$ & $110(13,7)$ \\
\hline \multicolumn{7}{|l|}{ Escolaridade } \\
\hline Ensino Fundamental incompleto & $40(33,1)$ & $39(32,3)$ & $27(22,3)$ & $38(31,4)$ & $45(37,2)$ & $49(40,5)$ \\
\hline Ensino Fundamental completo & $96(31,3)$ & $91(29,7)$ & $64(20,1)$ & $59(19,2)$ & $59(19,3)$ & $67(21,9)$ \\
\hline Ensino Médio completo & $179(24,7)$ & $173(23,8)$ & $114(15,7)$ & $98(13,5)$ & $97(13,3)$ & $125(17,2)$ \\
\hline Ensino Superior completo/ & $226(22,3)$ & $233(23,0)$ & $153(15,1)$ & $101(10,0)$ & $91(9,0)$ & $125(12,3)$ \\
\hline \multicolumn{7}{|l|}{ Pós-graduação } \\
\hline \multicolumn{7}{|l|}{ Transtorno mental comum } \\
\hline Positivo & $247(34,7)$ & $251(35,2)$ & $176(24,7)$ & $169(23,7)$ & $171(24,0)$ & $206(28,9)$ \\
\hline Negativo & $294(20,1)$ & $284(19,5)$ & $182(12,5)$ & $127(8,7)$ & $120(8,2)$ & $158(10,8)$ \\
\hline \multicolumn{7}{|l|}{$\begin{array}{l}\text { Evento de vida produtor de } \\
\text { estresse }\end{array}$} \\
\hline Presente & $444(27,7)$ & $442(27,5)$ & $299(18,6)$ & $253(15,8)$ & $250(15,6)$ & $313(19,5)$ \\
\hline Ausente & $102(17,4)$ & $97(16,6)$ & $61(10,4)$ & $46(7,9)$ & $45(7,7)$ & $56(9,5)$ \\
\hline \multicolumn{7}{|l|}{ Apoio social } \\
\hline Baixo + médio & $401(27,7)$ & $381(26,3)$ & $257(17,8)$ & $238(16,4)$ & $230(15,9)$ & $292(20,2)$ \\
\hline Alto & $126(18,6)$ & $136(20,1)$ & $90(13,3)$ & $52(7,7)$ & $52(7,7)$ & $63(9,3)$ \\
\hline
\end{tabular}

* Todas as diferenças foram estatisticamente significativas, valor de $p<0,01$.

prevalência de queixa frequente é de 20,3\% para a dificuldade em iniciar o sono, $17,7 \%$ para a dificuldade em manter o sono e $22,4 \%$ para a queixa geral de insônia.

Observa-se um comportamento similar entre as variáveis de desfecho - dificuldade em iniciar o sono, em manter o sono ou queixa geral de insônia - sendo a diferença encontrada na intensidade desses desfechos, ou seja, se a insônia sofrida foi ocasional ou frequente.

Em todos os desfechos analisados, observa-se que as mulheres na menopausa há menos de 60 meses apresentaram uma redução acentuada na magnitude dos OR após o ajuste pelas variáveis socioeconômicas e demográficas (idade, renda per capita e escolaridade), independente da frequência de insônia (ocasional ou frequente). Es- ses valores mantiveram uma redução contínua nas suas magnitudes conforme introduzimos, no modelo, as variáveis psicossociais (transtornos mentais comuns, eventos de vida produtores de estresse e apoio social).

Em comparação, as mulheres na menopausa há mais de 60 meses também apresentaram uma acentuada redução dos OR após o primeiro modelo de ajuste, independente da frequência da insônia, porém, diferentemente do grupo anterior, quando introduzimos, no modelo, as variáveis psicossociais, os valores dos OR não sofrem mudanças significativas. Podemos observar que as análises brutas mostraram que mulheres na menopausa não recente apresentavam uma chance de insônia frequente em torno de duas vezes maior do que a de mulheres que estavam 
Prevalência e odds ratios (OR) brutos e ajustados e respectivos intervalos de $95 \%$ de confiança (IC95\%) da associação entre dificuldade em iniciar o sono, dificuldade em manter o sono e queixa de insônia frequente e estado de menopausa ( $n=2.190)$. Estudo Pró-Saúde, 1999.

\begin{tabular}{|c|c|c|c|c|c|c|}
\hline & \multicolumn{6}{|c|}{ Queixa frequente } \\
\hline & n (\%) & $\begin{array}{l}\text { OR bruto } \\
\text { (IC95\%) }\end{array}$ & $\begin{array}{c}\text { Modelo } 1 \\
\text { (IC95\%) }\end{array}$ & $\begin{array}{c}\text { Modelo } 2 \\
\text { (IC95\%) }\end{array}$ & $\begin{array}{c}\text { Modelo } 3 \\
\text { (IC95\%) }\end{array}$ & $\begin{array}{c}\text { Modelo } 4 \\
\text { (IC95\%) }\end{array}$ \\
\hline \multicolumn{7}{|l|}{ Dificuldade em iniciar o sono } \\
\hline \multicolumn{7}{|l|}{ Menopausa } \\
\hline Não & $206(11,8)$ & 1,00 & 1,00 & 1,00 & 1,00 & 1,00 \\
\hline Recente (< 60 meses) & $39(20,3)$ & $2,12(1,42-3,15)$ & $1,39(0,86-2,25)$ & $1,24(0,75-2,05)$ & $1,37(0,84-2,23)$ & $1,19(0,72-1,98)$ \\
\hline Não recente (+ 60 meses) & $54(20,9)$ & $2,31(1,62-3,28)$ & $1,53(0,95-2,46)$ & $1,51(0,92-2,48)$ & $1,56(0,96-2,52)$ & $1,53(0,92-2,52)$ \\
\hline \multicolumn{7}{|l|}{ Dificuldade em manter o sono } \\
\hline \multicolumn{7}{|l|}{ Menopausa } \\
\hline Não & $197(11,3)$ & 1,00 & 1,00 & 1,00 & 1,00 & 1,00 \\
\hline Recente ( $<60$ meses) & $34(17,7)$ & $1,94(1,28-2,95)$ & $1,22(0,73-2,04)$ & $1,07(0,62-1,83)$ & $1,22(0,72-2,04)$ & $1,05(0,61-1,80)$ \\
\hline Não recente (+ 60 meses) & $64(24,8)$ & $2,77(1,98-3,87)$ & $1,86(1,16-2,97)$ & $1,82(1,11-2,98)$ & $1,86(1,15-2,99)$ & $1,81(1,09-2,98)$ \\
\hline \multicolumn{7}{|l|}{ Queixa geral de insônia } \\
\hline \multicolumn{7}{|l|}{ Menopausa } \\
\hline Não & $255(14,7)$ & 1,00 & 1,00 & 1,00 & 1,00 & 1,00 \\
\hline Recente ( $<60$ meses) & $43(22,4)$ & $1,82(1,25-2,65)$ & $1,30(0,83-2,05)$ & $1,62(0,72-1,87)$ & $1,28(0,81-2,06)$ & $1,12(0,70-1,82)$ \\
\hline Não recente (+ 60 meses) & $71(27,5)$ & $2,37(1,73-3,24)$ & $1,73(1,12-2,66)$ & $1,70(1,08-2,67)$ & $1,75(1,13-2,72)$ & $1,71(1,08-2,73)$ \\
\hline
\end{tabular}

Modelo 1: ajustado por renda per capita, escolaridade e idade; Modelo 2: ajustado por renda per capita, escolaridade, idade e transtorno mental comum; Modelo 3: ajustado por renda per capita, escolaridade, idade e apoio social; Modelo 4: ajustado por renda per capita, escolaridade, idade, transtorno mental comum, eventos de vida produtores de estresse e apoio social.

Nota: Todas as diferenças foram estatisticamente significativas, valor de $p<0,01$.

na menopausa há menos de 60 meses (OR variando de 2,31 a 2,77). Após ajuste por variáveis socioeconômicas, demográficas, transtorno mental comum, eventos de vida produtores de estresse e apoio social, houve redução na magnitude dos OR, sendo esses OR = 1,53 (IC95\%: 0,92-2,52) para dificuldade em iniciar o sono, OR $=1,81$ (IC95\%: 1,09-2,98) para dificuldade em manter o sono e OR = 1,71 (IC95\%: 1,08-2,73) para queixa geral de insônia.

\section{Discussão}

As análises não ajustadas mostraram uma chance em torno de duas vezes maior de insônia frequente entre mulheres com mais de 60 meses de amenorreia.

A redução importante na magnitude dos OR após os ajustes pelas variáveis socioeconômicas e demográficas reforça a importância de tais variáveis como confundidoras na associação entre a menopausa e a insônia. Entretanto, observouse que as variáveis psicossociais revelaram-se como potenciais mediadoras dessa associação, principalmente entre as mulheres que estão na menopausa há menos de 60 meses.
Estudo realizado na Holanda mostrou uma chance duas vezes maior de apresentar insônia em mulheres na pós-menopausa, quando comparadas a mulheres fora desse período 28 . Entretanto, este estudo não deixou claro em que sentido essa associação poderia ser considerada independente, já que, apesar dos resultados decorrentes de análise ajustada, não foram descritas quais as variáveis incluídas no modelo. Já o estudo de Shin et al. 29 analisou separadamente mulheres na peri e pós-menopausa e observou chances de 2,1 e 1,4 para insônia, após ajuste por depressão e idade. A comparação desses resultados com os nossos deve ser feita com cautela, já que os ajustes nos dois estudos não se referem exatamente às mesmas variáveis. Estudo que avaliou a qualidade do sono (definida como dormir mal sempre ou a maior parte das vezes) no climatério, em São Paulo, Brasil, encontrou associação entre peri e pós-menopausa cirúrgica com dormir mal quando comparadas à pré-menopausa, com OR = 2,63 (IC95\%: 1,25-5,51) e OR = 2,78 (IC95\%: 1,18-6,60), respectivamente ${ }^{3}$. As prevalências da queixa de dificuldade de iniciar o sono, de manter o sono e da queixa geral de insônia mais baixas no nosso estudo em relação aos achados na literatura 3,30,31 podem ser decor- 
Prevalência e odds ratios (OR) brutos e ajustados e respectivos intervalos de $95 \%$ de confiança (IC95\%) da associação entre dificuldade em iniciar o sono, dificuldade em manter o sono e queixa de insônia ocasional e estado de menopausa ( $n=2.190)$. Estudo Pró-Saúde, 1999.

\begin{tabular}{|c|c|c|c|c|c|c|}
\hline & \multicolumn{6}{|c|}{ Queixa ocasional } \\
\hline & n (\%) & $\begin{array}{l}\text { OR bruto } \\
\text { (IC95\%) }\end{array}$ & $\begin{array}{c}\text { Modelo } 1 \\
\text { (IC95\%) }\end{array}$ & $\begin{array}{c}\text { Modelo } 2 \\
\text { (IC95\%) }\end{array}$ & $\begin{array}{c}\text { Modelo } 3 \\
\text { (IC95\%) }\end{array}$ & $\begin{array}{c}\text { Modelo } 4 \\
\text { (IC95\%) }\end{array}$ \\
\hline \multicolumn{7}{|l|}{ Dificuldade em iniciar o sono } \\
\hline \multicolumn{7}{|l|}{ Menopausa } \\
\hline Não & $416(23,9)$ & 1,00 & 1,00 & 1,00 & 1,00 & 1,00 \\
\hline Recente ( $<60$ meses) & $53(27,6)$ & $1,42(1,00-2,02)$ & $1,32(0,88-1,98)$ & $1,22(0,80-1,84)$ & $1,24(0,82-1,87)$ & $1,13(0,74-1,72)$ \\
\hline Não recente (+ 60 meses) & $77(29,8)$ & $1,63(1,20-2,21)$ & $1,56(1,05-2,33)$ & $1,58(1,04-2,36)$ & $1,50(1,0-2,25)$ & $1,51(0,99-2,29)$ \\
\hline \multicolumn{7}{|l|}{ Dificuldade em manter o sono } \\
\hline \multicolumn{7}{|l|}{ Menopausa } \\
\hline Não & $419(24,1)$ & 1,00 & 1,00 & 1,00 & 1,00 & 1,00 \\
\hline Recente ( $<60$ meses) & $58(30,2)$ & $1,55(1,10-2,19)$ & $1,31(0,89-1,93)$ & $1,20(0,80-1,81)$ & $1,26(0,85-1,88)$ & $1,15(0,76-1,74)$ \\
\hline Não recente (+ 60 meses) & $62(24,0)$ & $1,26(0,91-1,74)$ & $1,09(0,72-1,64)$ & $1,13(0,74-1,72)$ & $1,04(0,69-1,58)$ & $1,08(0,70-1,66)$ \\
\hline \multicolumn{7}{|l|}{ Queixa geral de insônia } \\
\hline \multicolumn{7}{|l|}{ Menopausa } \\
\hline Não & $278(16,0)$ & 1,00 & 1,00 & 1,00 & 1,00 & 1,00 \\
\hline Recente ( $<60$ meses) & $37(19,3)$ & $1,43(0,97-2,13)$ & $1,40(0,89-2,19)$ & $1,27(0,80-2,02)$ & $1,30(0,82-2,05)$ & $1,17(0,73-1,88)$ \\
\hline Não recente (+ 60 meses) & $45(17,4)$ & $1,38(0,96-1,97)$ & $1,34(0,85-2,13)$ & $1,39(0,87-2,23)$ & $1,32(0,83-2,11)$ & $1,37(0,85-2,21)$ \\
\hline
\end{tabular}

Modelo 1: ajustado por renda per capita, escolaridade e idade; Modelo 2: ajustado por renda per capita, escolaridade, idade e transtorno mental comum Modelo 3: ajustado por renda per capita, escolaridade, idade e apoio social; Modelo 4: ajustado por renda per capita, escolaridade, idade, transtorno mental comum, eventos de vida produtores de estresse e apoio social.

Nota: Todas as diferenças foram estatisticamente significativas; valor de $p<0,01$

rentes de diferenças nas metodologias utilizadas, em particular em relação às definições de insônia e às populações de estudo. A utilização de critérios mais abrangentes na definição de insônia pode contribuir para aumentar a estimativa da prevalência. Em nosso estudo, verificamos que a prevalência de queixa de insônia aumentava consideravelmente dependendo de onde alocávamos as mulheres que respondiam "às vezes" para as perguntas sobre dificuldade em iniciar o sono ou em manter o sono. Decidimos manter um critério mais restritivo e criamos uma categoria para avaliar essas mulheres (insônia ocasional). Outra possível explicação para as prevalências mais baixas encontradas no nosso estudo é que nosso universo é composto por funcionárias públicas, com estabilidade no emprego, nível de escolaridade e renda superiores ao da média populacional, fatores esses inversamente relacionados à insônia 32,33,34,35.

Uma limitação metodológica importante é que, por ser constituída por funcionárias de uma universidade pública, a nossa amostra de estudo se diferencia da população geral, por possuir estabilidade no emprego e um patamar socioeconômico mais elevado. Sabe-se que, entre desem- pregadas, com nível socioeconômico mais baixo ou instabilidade no emprego, a ocorrência de eventos de vida estressantes, transtornos mentais e insônia são mais frequentes 36 , de forma que não há como garantir a generalização desses resultados a diferentes grupos ou populações.

Um outro limitador é a forma de mensurar a insônia, já que nossos dados são obtidos a partir de questionários autopreenchíveis. E estudos demonstram uma diferença entre dados objetivos (obtidos com polissonografia) e subjetivos (questionários ou entrevistas) na mensuração de insônia em mulheres na menopausa 1,37,38. Os resultados devem ser comparados com cautela, sempre observando as diferenças metodológicas, de amostra, definições de insônia e menopausa, utilizadas pelos diferentes estudos 39 .

A qualidade do sono é um importante determinante para a saúde e a qualidade de vida em geral $40,41 \mathrm{e}$, em particular, entre as mulheres na menopausa 3 . Segundo Cheng et al. ${ }^{42}$, os distúrbios do sono podem estar relacionados a questões hormonais, como a falta de estrógeno. No entanto, os próprios autores ressaltam que esses dados não são conclusivos, já que outros estudos sugerem que os distúrbios do sono não de- 
pendem do estado de menopausa. Em conjunto com o estado de menopausa, numerosos fatores podem contribuir para a insônia em mulheres de meia-idade, entre eles, fatores sociais 12,16,18, condições psiquiátricas $1,2,6,10,13$, sintomas vasomotores 5,6 , terapia hormonal, peso corporal ou outros transtornos do sono 42.

Nossos resultados mostram que a menopausa não recente associou-se, de forma importante, com a presença de problemas relacionados ao sono que se manifestaram em todos os desfechos estudados - dificuldade de iniciar o sono, de manter o sono e queixa de insônia - independente de fatores socioeconômicos, demográficos e psicossociais, sugerindo independência dessa associação em relação a outros fatores também prevalentes nessa fase da vida e também associados com a menopausa.
Cabe ressaltar que a relação entre insônia e fatores psicossociais pode ser bidirecional, ou seja, a insônia tanto pode ser um fator de risco 43 quanto uma consequência de transtornos mentais comuns, da depressão 43,44 , de distúrbios de ansiedade, do abuso de álcool 45 e de alguns eventos de vida produtores de estresse 17 . Nesse sentido, se não tratada devidamente, a insônia pode favorecer o aparecimento de tais agravos, independentemente da idade ou do status de menopausa da mulher.

Nossos resultados revelam a importância no manejo e no tratamento da insônia associados à menopausa, principalmente se considerarmos que a maioria das mulheres que sofrem de insônia nessa fase da vida não são diagnosticadas, tampouco tratadas.

\section{Resumen}

En este estudio se evaluó la asociación entre insomnio y menopausia y la influencia de las variables socioeconómicas y psicosociales, en un estudio transversal con 2.190 mujeres de una universidad (Estudio Pro-Salud), a partir de un cuestionario autoadministrado con variables de la menopausia, insomnio, trastornos mentales, situaciones de estrés vital, apoyo social y variables socioeconómicas. Se calcularon los odds ratio mediante regresión logística multivariante con desenlace politómico. Después de ajustar por factores de confusión sociodemográficos potenciales, las mujeres menopáusicas desde hace más de 60 meses fueron más propensas a reportar quejas frecuentes de sueño (OR entre 1,53 y
1,86) que las menopáusicas hace menos de 60 meses Después de los ajustes, en el primer grupo, para las variables psicosociales la magnitud de los OR se redujo a 1,53 (IC95\%: 0,92-2,52) para la dificultad para iniciar el sueño, un 1,81 (IC95\%: 1,09-2,98) para mantener el sueño y un 1,71 (IC95\%: 1,08-2,73) para las quejas de insomnio en general. Los factores psicosociales pueden mediar en la manifestación del insomnio en las mujeres menopáusicas.

Insomnia; Transtornos del Sueño; Menopausia 


\section{Colaboradores}

J. R. Robaina revisou a literatura, realizou a análise estatística dos dados, a redação, a interpretação e a discussão dos resultados. C. S. Lopes participou do planejamento e da implementação do Estudo Pró-Saúde e orientou a revisão bibliográfica, a análise de dados, a interpretação e a discussão dos resultados. L. Rotenberg orientou a revisão bibliográfica, a análise de dados e a interpretação e a discussão dos resultados. E. Faerstein participou do planejamento e da implementação do Estudo Pró-Saúde, da interpretação e da discussão dos resultados. Todos os autores trabalharam na elaboração e na revisão final do artigo.

\section{Referências}

1. Campos HH, Bittencourt LRA, Haidar MA, Tufik S, Baracat EC. Prevalência de distúrbios do sono na pós-menopausa. Rev Bras Ginecol Obstet 2005; 27:731-6.

2. Pérez JAM, Garcia FC, Palacios S, Pérez M. Epidemiology of risk factors and symptoms associated with menopause in Spanish women. Maturitas 2009; 62:30-6.

3. Souza CL, Aldrighi JM, Lorenzi Filho G. Qualidade do sono em mulheres paulistanas no climatério. Rev Assoc Med Bras (1992) 2005; 51:170-6.

4. Hachul H, Brandão LC, Bittencourt LRA, D'Almeida V, Andersen ML, Baracat EC, et al. Clinical profile of menopausal insomniac women referred to sleep laboratory. Acta Obstet Gynecol Scand 2009; 88:422-7.

5. Pedro AO, Pinto-Neto AM, Costa-Paiva LHS, Osis MJD, Hardy EE. Síndrome do climatério: inquérito populacional domiciliar em Campinas, SP. Rev Saúde Pública 2003; 37:735-42.

6. Landis CA, Moe KE. Sleep and menopause. Nurs Clin North Am 2004; 39:97-115.

\section{Agradecimentos}

A toda a equipe do Estudo Pró-Saúde, que participou das etapas de trabalho de campo e digitação dos dados, e aos funcionários técnicos administrativos da universidade que participaram da pesquisa.

\section{Conflito de interesses}

O presente estudo não apresenta conflito de interesse por nenhum de seus autores.
7. Regestein QR, Friebely J, Shifren JL, Scharf MB, Wiita B, Carver J, et al. Self-reported sleep in postmenopausal women. Menopause 2004; 11:198207.

8. Kalleinen N. Sleep and menopause - hormone therapy and sleep deprivation [Tese de Doutorado]. Turku: University of Turku; 2008.

9. Utian WH. Menopause - a modern perspective from a controversial history. Maturitas 1997; 26:7382.

10. Amore M, Di Donato P, Berti A, Palareti A, Chirico C, Papalini A, et al. Sexual and psychological symptoms in the climacteric years. Maturitas 2007; 56:303-11.

11. Roberts RE. Positive well-being and sleep. J Psychosom Res 2008; 64:417-8.

12. Basta M, Chrousos GP, Vela-Bueno A, Vgontzas AN. Chronic insomnia and stress system. Sleep Med Clin 2007; 2:279-91. 
13. Strauss JR. The reciprocal relationship between menopausal symptoms and depressive symptoms: a 9-year longitudinal study of American women in midlife. Maturitas 2011; 70:302-6.

14. Amore M, Di Donato P, Papalini A, Berti A, Palareti A, Ferrari G, et al. Psychological status at the menopausal transition: an Italian epidemiological study. Maturitas 2004; 48:115-24.

15. Terauchi M, Hiramitsu S, Akiyoshi M, Owa Y, Kato K, Obayashi S, et al. Associations among depression, anxiety and somatic symptoms in peri- and postmenopausal women. J Obstet Gynaecol Res 2013; 39:1007-13.

16. Gath D, Osborn M, Bungay G, Iles S, Day A, Bond A, et al. Psychiatric disorder and gynaecological symptoms in middle aged women: a community survey. Br Med J (Clin Res Ed) 1987; 294:213-8.

17. Robaina JR, Lopes CS, Rotenberg L, Faerstein E, Fischer FM, Moreno CRC, et al. Eventos de vida produtores de estresse e queixas de insônia entre auxiliares de enfermagem de um hospital universitário no Rio de Janeiro: Estudo Pró-Saúde. Rev Bras Epidemiol 2009; 12:501-9.

18. Pimenta F, Leal I, Maroco J, Ramos C. Menopausal symptoms: do life events predict severity of symptoms in peri- and post-menopause? Maturitas 2012; 72:324-31.

19. Faerstein E, Lopes CS, Valente K, Plá MAS, Ferreira MB. Pré-testes e um questionário multidimensional autopreenchível: a experiência do Estudo Pró-Saúde UERJ. Physis (Rio J.) 1999; 9:117-30.

20. Ohayon MM. Epidemiology of insomnia: what we know and what we still need to learn. Sleep Med Rev 2002; 6:97-111.

21. Goldberg EL, Blackwell B. The detection of psychiatric illness by questionnaire. Maudsley monograph 21. London: Oxford University Press; 1972.

22. Wall RI, Bolden CS, Borri AJ. Minor psychiatric disorder in NHS trust staff: occupational and gender differences. Br J Psychiatry 1997; 171:519-23.

23. Goldberg DP, Williams P. The user's guide to the General Health Questionnaire. Windsor: Nfer-Nelson; 1988.

24. Lopes CS, Faerstein E. Confiabilidade do relato de eventos de vida estressantes em um questionário autopreenchido: Estudo Pró-Saúde. Rev Bras Psiquiatr 2001; 23:126-33.

25. Lopes CS, Faerstein E, Chor D. Eventos de vida produtores de estresse e transtornos mentais comuns: resultados do Estudo Pró-Saúde. Cad Saúde Pública 2003; 19:1713-20

26. Sherbourne CD, Stewart AL. The MOS Social Support Survey. Soc Sci Med 1991; 38:705-14.

27. Griep RH, Chor D, Faerstein E, Lopes C. Apoio social: confiabilidade teste-reteste de escala no Estudo Pró-Saúde. Cad Saúde Pública 2003; 19:625-34.

28. Maartens LW, Leusink GL, Knottnerus JA, Smeets CG, Pop VJ. Climacteric complaints in the community. Fam Pract 2001; 18:189-94.

29. Shin C, Lee S, Lee T, Shin K, Yi H, Kim K, et al. Prevalence of insomnia and its relationship to menopausal status in middle-aged Korean women. Psychiatry Clin Neurosci 2005; 59:395-402.
30. Blümel JE, Cano A, Mezones-Holguín E, Barón G, Bencosme A, Benítez Z, et al. A multinational study of sleep disorders during female mid-life. Maturitas 2012; 72:359-66.

31. Hachul H, Bittencourt LRA, Soares Jr. JM, Tufik S, Baracat EC. Sleep in post-menopausal women: differences between early and late post-menopause Eur J Obstet Gynecol Reprod Biol 2009; 145:81-4.

32. Bixler EO, Kales A, Soldatos CR, Kales JD, Healey S. Prevalence of sleep disorders in the Los Angeles Metropolitan Area. Am J Psychiatry 1979; 136:1257-62.

33. Pallensen S, Nordhus IH, Nielsen H, Havik OE, Kvale G, Johnsen BH, et al. Prevalence of insomnia in the adult Norwegian population. Sleep 2001; 24:771-9.

34. Li RHY, Ho SC, Fong SYY. Gender differences in insomnia - a study in the Hong Kong Chinese population. J Psychosom Res 2002; 53:601-9.

35. Lopes CS, Robaina JR, Rotenberg L. Epidemiology of insomnia: prevalence and risk factors In: Sahoo S, editor. Can't sleep? Issues of being an insomniac. Rijeka: InTech; 2012. p. 3-22.

36. Kim K, Uchiyama M, Okawa M, Liu X, Ogihara R. An epidemiological study of insomnia among the Japanese general population. Sleep 2000; 23:41-7.

37. Kalleinen N, Polo-Kantola P, Himanen SL, Alhola P, Joutsen A, Urrila AS, et al. Sleep and the menopause - do postmenopausal women experience worse sleep than premenopausal women? Menopause Int 2008; 14:97-104.

38. Xu M, Bélanger L, Ivers H, Guay B, Zhang J, Morin CM. Comparison of subjective and objective sleep quality in menopausal and non-menopausal women with insomnia. Sleep Med 2011; 12:65-9.

39. Vitiello MV, Larsen LH, Moe KE. Age-related sleep change: gender and estrogen effects on the subjective-objective sleep quality relationships of healthy, noncomplaining older men and women. J Psychosom Res 2004; 56:503-10.

40. Rocha FL, Costa MFFL. Epidemiologia e impacto dos distúrbios do sono. J Bras Psiquiatr 2000; 49:167-80.

41. Schubert CR, Cruickshanks KJ, Dalton DS, Klein BEK, Klein R, Nondahl DM. Prevalence of sleep problem and quality of life in an older population. Sleep 2002; 25:48-52.

42. Cheng MH, Hsu CY, Wang SJ, Lee SJ, Wang PH, Fuh JL. The relationship of self-reported sleep disturbance, mood, and menopause in a community study. Menopause 2008; 15:958-62.

43. Lustberg L, Reynolds CF. Depression and insomnia: questions of cause and effect. Sleep Med Rev 2000; 4:253-62.

44. Roberts RE, Shema SJ, Kaplan GA, Strawbridge WJ. Sleep complaints and depression in an aging cohort: a prospective perspective. Am J Psychiatry 2000; 157:81-8.

Recebido em 21/Mar/2014

Versão final reapresentada em 25/Set/2014

Aprovado em 01/Out/2014 